MAGNETOHYDRODYNAMICS Vol. 54 (2018), No. 1-2, pp. 97-101

DOI: $10.22364 / \mathrm{mhd} .54 .1-2.17$

\title{
FORMATION OF BRIDGES BETWEEN NON-MAGNETIC SURFACES VIA THE MAGNETIC FLUID SURFACE DEFORMATION IN NON-UNIFORM MAGNETIC FIELDS
}

\author{
D. Pelevina, O. Sharova, A. Vinogradova, V. Turkov, V. Naletova \\ Lomonosov Moscow State University, Leninskie Gory, Moscow 119991, Russia
}

\begin{abstract}
Static shapes of the magnetic fluid surface in non-uniform magnetic fields are studied theoretically. In particular, the case when the magnetic fluid volume can fill the space between non-magnetic surfaces is addressed. Two typical non-uniform field geometries with spherical and cylindrical symmetries are analyzed, i.e. the field of a magnetizable sphere exposed to a uniform magnetic field and the field of a straight current-carrying wire.
\end{abstract}

Introduction. The surface of magnetic fluid (MF) can be deformed by a non-uniform magnetic field. This feature is used to develop different devices which can sustain a pressure drop, such as valves [1], pumps [1-3], sensors [4], seals [5], in which the MF location is controlled by the magnetic field induced by a permanent magnet or by a coil. In this paper, the magnetic field of a magnetizable sphere in a uniform magnetic field and the magnetic field of a current-carrying wire are proposed e here to control the surface of the magnetic fluid which can fill the space between non-magnetic surfaces. Two axially symmetric problems of the MF equilibrium are solved theoretically.

1. General equations. The equilibrium equation for a heavy, incompressible, homogenous, isothermal MF with the axially symmetric surface $z=h(r)$ and dynamic boundary condition have the following form:

$$
-\nabla^{j} p_{i j} \mathbf{e}^{i}+\rho_{\mathrm{f}} \mathbf{g}=0, \quad\left[p_{i j} \mathbf{e}^{i} n^{j}\right]_{\mathrm{f}}^{s}= \pm 2 \sigma K \mathbf{n} .
$$

The signs "+" ("-") should be chosen when a surrounding non-magnetic fluid (NMF) with the magnetic permeability $\mu_{\mathrm{s}}=1$ is placed above (beneath) the MF. Here, 'f', 's' are the indices corresponding, respectively, to MF and NMF, $\mathbf{e}^{i}$ denotes the basis vectors, $\mathbf{g}$ is the gravitational acceleration, $\mathbf{n}$ is the external normal vector, $\rho_{\mathrm{f}}$ is the MF density, $2 K=\left(h^{\prime \prime}+h^{\prime 3} / r+h^{\prime} / r\right) /\left(1+h^{\prime 2}\right)^{3 / 2}$ is the mean curvature of $z=h(r), \sigma$ is the constant coefficient of surface tension. The stress tensor is

$$
p_{i j}=-p^{H} g_{i j}+\frac{H_{i} B_{j}}{4 \pi}-\frac{H^{2} g_{i j}}{8 \pi}=-p g_{i j}+\frac{H_{i} B_{j}}{4 \pi}-\frac{H B g_{i j}}{8 \pi}, p=p^{H}-\frac{M H}{2} .
$$

Later, the isopycnal case is considered: $\rho_{\mathrm{f}}=\rho_{\mathrm{s}}$.

2. Magnetizable sphere in a uniform magnetic field. Let us consider the equilibrium of a magnetizable sphere (radius $R_{\mathrm{b}}$, mass $M_{\mathrm{b}}$, volume $V_{\mathrm{b}}$ ) partially immersed into the MF volume on the horizontal plane under the action of gravity subject to a uniform vertical magnetic field $\mathbf{H}_{\infty}=\left(0,0, H_{\infty}\right)$ (see Fig. 1a). We introduce a cylindrical coordinate system $(r, \varphi, z)$ with its origin in the sphere center; the axis $z$ is directed vertically. The following assumptions are made: i) a non-inductive approximation, $\mu_{\mathrm{f}}-1 \ll 1$ (hence, $H \approx H_{0}, H_{0}$ is the magnetic field around the sphere without MF in the applied field $H_{\infty}$ ); ii) there is no surface tension, $\sigma=0$; iii) $\mu_{\mathrm{f}}=$ const (the volume magnetic force is zero). 
(a)
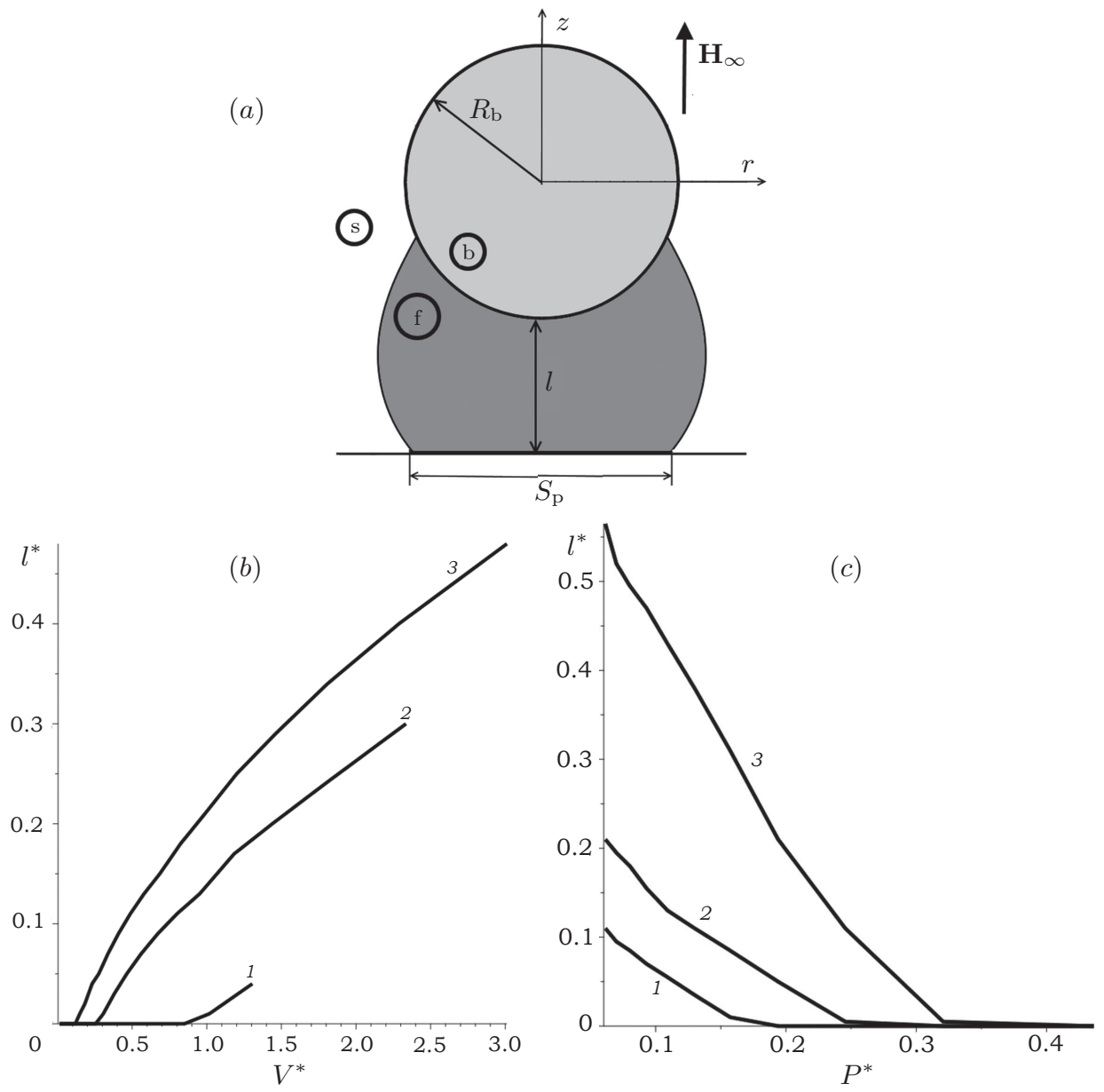

Fig. 1. (a) A magnetizable sphere in the MF volume in a vertical magnetic field; (b) dependence of $l^{*}$ on $V^{*}$ for $1-P^{*}=0.24\left(H_{\infty}=400 \mathrm{Oe}\right), 2-P^{*}=0.11\left(H_{\infty}=600 \mathrm{Oe}\right)$, $3-P^{*}=0.06\left(H_{\infty}=800 \mathrm{Oe}\right) ;(c)$ dependence of $l^{*}$ on $P^{*}$ for $1-V^{*}=0.5,2-V^{*}=1,3$ $-V^{*}=4$.

From Eqs. (1) we obtain pressure values in MF and in NMF: $p_{\mathrm{f}, \mathrm{s}}=p_{0 \mathrm{f}, \mathrm{s}}-$ $\rho_{\mathrm{f}, \mathrm{s}} g z, p_{0 \mathrm{f}, \mathrm{s}}=$ const, and the dynamic boundary condition (1) has the following form:

$$
0=p_{n n}^{(\mathrm{s})}-p_{n n}^{(\mathrm{f})} \approx p_{0 \mathrm{f}}-p_{0 \mathrm{~s}}+\left(\mu_{\mathrm{f}}-1\right) H_{\mathrm{c}}^{2} /(8 \pi) .
$$

From Eq. (2) it follows that the MF surface $z=h(r)$ coincides with the curve of the steady magnetic field: $H_{0}(r, z)=H_{\mathrm{c}}=$ const.

The surface force $\mathbf{F}$ acting on the sphere is calculated as

$$
\mathbf{F}=\int_{S_{\mathrm{b}}} p_{i j} n_{\mathrm{b}}^{j} \mathbf{e}^{i} \mathrm{~d} s=\frac{\mu_{\mathrm{f}}-1}{8 \pi} \int_{S_{\mathrm{p}}}\left(H_{0}^{2}-H_{\mathrm{c}}^{2}\right) \mathbf{e}_{\mathbf{z}} \mathrm{d} s .
$$

Here $S_{\mathrm{b}}$ is the sphere contact surface, $S_{\mathrm{p}}$ is the contact area of the MF and the plane. The expression for the magnetic field magnitude $H_{0}$ is the following

$$
H_{0}=H_{\infty} \sqrt{\left(1+\frac{D}{\left(r^{2}+z^{2}\right)^{3 / 2}}-\frac{3 z^{2} D}{\left(r^{2}+z^{2}\right)^{5 / 2}}\right)^{2}+\frac{9 z^{2} r^{2} D^{2}}{\left(r^{2}+z^{2}\right)^{5}}}, D=R_{\mathrm{b}}^{3} \frac{\mu_{\mathrm{b}}-\mu_{\mathrm{s}}}{\mu_{\mathrm{b}}+2 \mu_{\mathrm{s}}} .
$$


Formation of bridges between non-magnetic surfaces via the magnetic fluid...

The condition of the sphere levitation in the MF volume is presented as

$$
\mathbf{P}+\mathbf{F}=0, \quad \mathbf{P}=\left(M_{\mathrm{b}}-\rho_{\mathrm{f}} V_{\mathrm{b}}\right) \mathbf{g},
$$

where $\mathbf{P}$ is the sphere weight. We introduce the following dimensionless parameters: $r^{*}=r / R_{\mathrm{b}}, z^{*}=z / R_{\mathrm{b}}, H^{*}=H_{0} / H_{\infty}$. The $z$-component of Eqs. (3) is

$$
P^{*}-\frac{1}{8 \pi} \int_{S_{\mathrm{b}}^{*}}\left(H_{0}^{* 2}-H_{\mathrm{c}}^{* 2}\right) \mathrm{d} s^{*}=0, \quad P^{*}=\frac{P}{H_{\infty}^{2} R_{\mathrm{b}}^{2}\left(\mu_{\mathrm{f}}-1\right)} .
$$

For the given MF volume $V^{*}$, the MF surface $h^{*}$ and the height $l^{*}$, on which the sphere levitates, can be determined from Eqs. (4). If $h^{*}$ begins at the point $\left(r_{0}^{*}, z_{0}^{*}\right)$ on the sphere surface, we can calculate the field $H_{\mathrm{c}}^{*}$ at this point. It should be noted that according to the type of $H_{0}$ curves, $h^{*}$ can begin only at $z_{0}^{*} \leq z_{c r}^{*}=-0.35$. Numerical simulations are made for the following parameters typical of the water-based ionic stabilized MFs: $R_{\mathrm{b}}=0.5 \mathrm{~cm}, M_{\mathrm{b}}=3.6 \mathrm{~g}, \mu_{\mathrm{f}}=1.3$, $\rho_{\mathrm{s}}=1.14 \mathrm{~g} / \mathrm{cm}^{3}$.

The dependences $l^{*}=l^{*}\left(V^{*}\right)$ and $l^{*}=l^{*}\left(P^{*}\right)\left(P^{*}\right.$ corresponds to $\left.H_{\infty}\right)$ are shown in Fig. $1 b$ and Fig. 1c, accordingly. It is found that for the given field $H_{\infty}$, there is the minimum volume $V_{\min }^{*}$ (the smallest volume at which the sphere levitates: $l^{*}>0$ ), the maximum volume $V_{\max }^{*}$ and the maximum height $l_{\max }^{*}$ (it corresponds to the maximum initial point $\left.z_{0}^{*}=z_{c r}^{*}\right)$.

With increasing $H_{\infty}$ (decreasing $P^{*}$ ), the sphere rises above the plane. Thus, in the presence of a plane above the sphere, the MF with the sphere can form a bridge between the non-magnetic surfaces and serve as a valve when the MF surface is deformed in the non-uniform magnetic field.

3. Straight current-carrying wire. An MF of volume $V$ between a cylindrical surface of radius $R_{\mathrm{c}}$ and two limiting right circular truncated conical surfaces with apex angles $\alpha_{1}$ and $\alpha_{2}$ (Fig. 2) is considered. The cones and the cylinder are coaxial, and a straight wire of radius $r_{0}$ carrying the current $I$ is located on its axis. The cones intersect in a circle of the wire radius $r_{0}$. We consider that i) the MF has an axially symmetric surface of revolution $z=h(r), r^{2}=x^{2}+y^{2}$ (the axis $z$ is directed along the axis of the wire). In this geometry, the magnetic field of the wire $\mathbf{H}$ is not deformed by the MF and $|\mathbf{H}|=H, H(r)=2 I /(\mathrm{cr})$, where $c$ is the speed of light in vacuum. ii) The MF does not wet the solid boundaries (the case of non-wetting), hence, $90^{\circ}<\theta_{1}, \theta_{2}, \theta_{3} \leq 180^{\circ}$, where $\theta_{1}$ and $\theta_{2}$ are, respectively, the wetting angles of the upper and of the lower cone, $\theta_{3}$ is the wetting angle of the cylinder. iii) The MF magnetization $M_{\mathrm{f}}$ is described by the Langevin law.

In this geometry, the MF bridge can sustain the pressure drop $\Delta p=p_{1}^{H}-p_{2}^{H}$. The pressure $p_{1}^{H}$ is maintained above the MF and the pressure $p_{2}^{H}$ is maintained under the MF. We further assume that $\Delta p \geq 0$. It should be noted that for $p_{1}^{H}=p_{2}^{H}$, the MF surface has a horizontal plane of symmetry $z=0$ and $r_{1}=r_{2}$ (see Fig. $2 a$, where $r_{1}\left(r_{2}\right)$ is the radius of points at which the upper (lower) contact surface of the fluids $h_{1}\left(h_{2}\right)$ intersects with the upper (lower) cone). For $p_{1}^{H}>p_{2}^{H}$, the MF goes downward along the axis $z$ and $r_{1}<r_{2}$ (see Figs. 2b,c), but the MF continues to contact simultaneously with the upper and lower cones. Using the assumptions mentioned above, from Eqs. (1) we can obtain a general analytical solution [6] which describes the MF surfaces $h_{1}$ and $h_{2}$.

A program was written in the computer simulation software Maple to determine the MF shape and its volume. We use the following dimensional parameters for the numeric calculations: $r_{0}=0.05 \mathrm{~cm}, R_{\mathrm{c}}=0.5 \mathrm{~cm}, \theta_{1}=\theta_{2}=\theta_{3}=175^{\circ}$, $\alpha_{1}=\alpha_{2}=5^{\circ}, \sigma=20 \mathrm{dyn} / \mathrm{cm}$, the MF temperature $T=300^{\circ} \mathrm{K}$, the MF saturation magnetization $M_{S}=56.6 \mathrm{G}$, the number of ferromagnetic particles per unit 
(a)
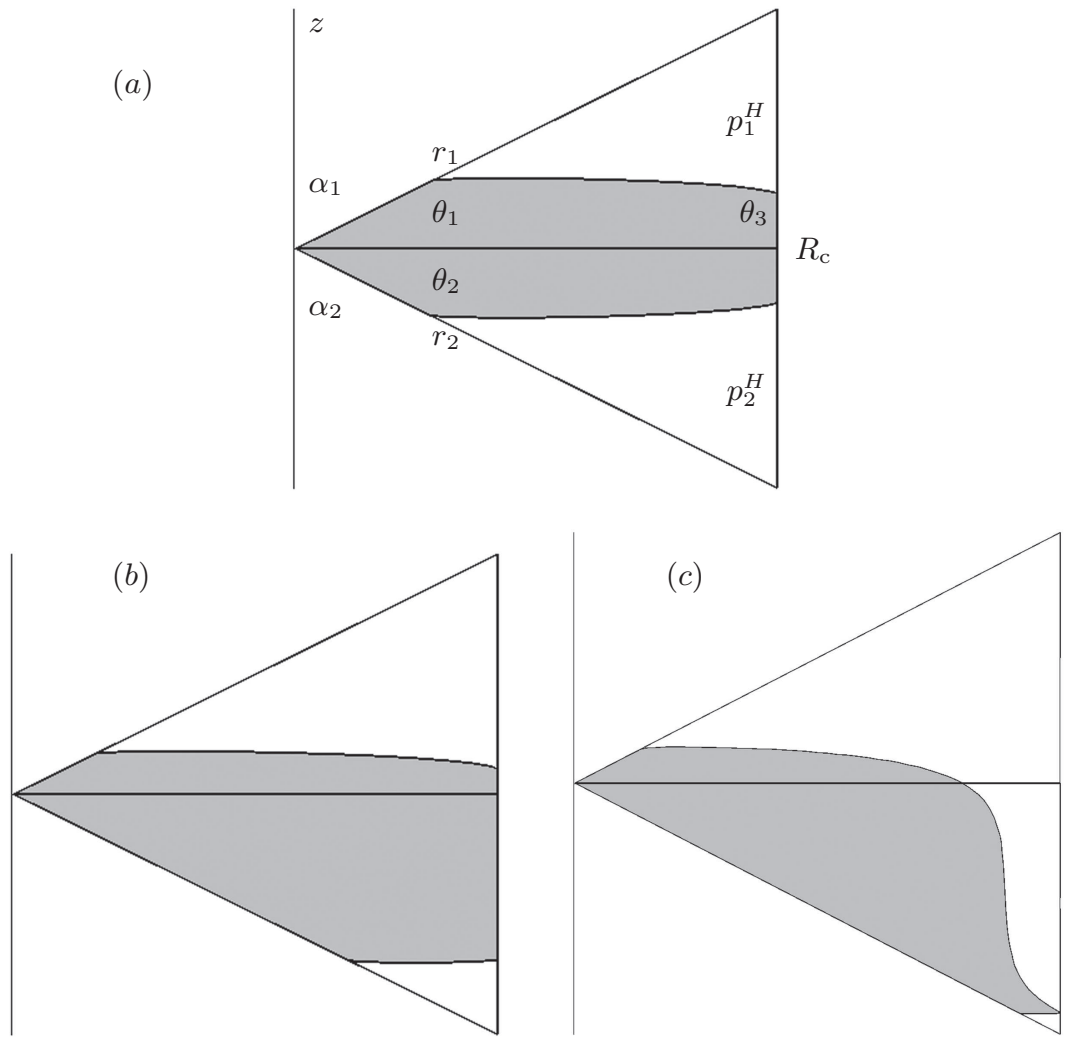

Fig. 2. Shapes of the fixed MF volume $V=2 \mathrm{~cm}^{3}$ at $I=53$ A for $(a) \Delta p=0 \mathrm{dyn} / \mathrm{cm}^{2}$; (b) $\Delta p=202.65 \mathrm{dyn} / \mathrm{cm}^{2}$; (c) $\Delta p=1013.25 \mathrm{dyn} / \mathrm{cm}^{2}$.

volume $n=1.9 \cdot 10^{17} \mathrm{~cm}^{-3}$. With the fixed volume $V=2 \mathrm{~cm}^{3}$, the MF shapes are illustrated in Fig. 2 at $I=53 \mathrm{~A}$ for different pressure drops $\Delta p$. The state (c) corresponds to the break situation when the MF volume is minimal to fill the space in the channel and the MF bridge opens the channel. The process $(a)-(c)$ in Fig. 2 can be cyclically repeated, so the MF bridge can serve as an MF valve or a fluid dispenser.

\section{Conclusions.}

- Two axially symmetric problems of the magnetic fluid equilibrium in nonuniform magnetic fields are solved theoretically in the isopycnal case.

- The equilibrium of a magnetizable sphere partially immersed into a magnetic fluid on the horizontal plane in a vertical uniform applied magnetic field is studied. The analytical expression for the magnetic force acting on the sphere from the magnetic fluid in the non-inductive approximation is found. The sphere can levitate in the magnetic fluid at a certain height. The dependences of the sphere levitation height on the magnetic fluid volume and on the applied magnetic field are obtained.

- Modelling of the magnetic fluid bridge equilibrium between two cones and a cylinder in the magnetic field of a straight current-carrying wire is performed when the magnetic fluid does not wet the surrounding solid boundaries. It is shown that the cones near the wire allow the magnetic fluid bridge to sustain 
Formation of bridges between non-magnetic surfaces via the magnetic fluid...

the pressure drop. The following cyclic process is possible: the pressure drop increases, the magnetic fluid bridge breaks up and opens the channel at a certain maximum pressure drop, the pressure drop decreases to zero and the magnetic fluid bridge shuts the channel.

- Thus, the magnetic fluid bridges and bodies in the magnetic fluid between non-magnetic surfaces can serve as valves, dispensers and interrupters, when the surface of magnetic fluid is deformed in non-uniform magnetic fields.

Acknowledgements.

This work was supported by RFBR grant No. 16-31-60091.

\section{References}

[1] H. Hartshorne, C.J. Backhouse, W.E. Lee. Ferrofluid-based microchip pump and valve. Sensors and Actuators B: Chemical, vol. 99 (2004), no. 2/3, pp. 592-600.

[2] C. Yamahata, M. Chastellain, V.K. Parashar, A. Petri, H. HofMANn, M.A.M. GiJs. Plastic micropump with ferrofluidic actuation. Journal of Microelectromechanical Systems, vol. 14 (2005), no. 1, pp. 96-102.

[3] K. Das, M. Sarkar, A. Mukhopadhyay, R. Ganguly. Transient response of a ferrofluid plug-driven micropump. Magnetohydrodynamics, vol. 49 (2013), no. $3 / 4$, pp. 499-504.

[4] J.-C. Bacri, J. Lenglet, R. Perzynski, J. Servais. Magnetic fluid pressure sensor. J. Magn. Magn. Mater, vol. 122 (1993), no. 1/3, pp. 399-402.

[5] B.M. BerkovskiI, M.S. Krakov and V.K. Rakhuba. Design problems and use limitations for magnetic-fluid seals. Magnetohydrodynamics, vol. 18 (1982), no. 1, pp. 68-74.

[6] A.S. Vinogradova, V.A. Naletova. Ferrofluid bridge between two cones and a cylinder in the magnetic field of a line conductor. In: Proc. the 9th PAMIR International Conference (Riga, Latvia, June 16-20, 2014), pp. 297301.

Received 27.12.2017 